\title{
Analysis of the extended stress-based forming limit curve considering the effects of strain path and through-thickness normal stress
}

\author{
Ramin Hashemi ${ }^{\mathrm{a}, \mathrm{b}, \mathrm{c}, *}$, Karen Abrinia ${ }^{\mathrm{c}}$ \\ a Faculty of Mechanical Engineering, KN Toosi University of Technology, P.O. Box 19395-1999, Tehran, Iran \\ ${ }^{\mathrm{b}}$ School of Mechanical Engineering, Iran University of Science and Technology, Tehran, Iran

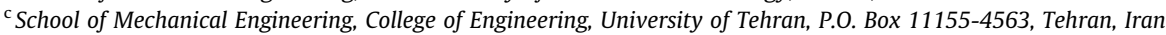

\section{A R T I C L E I N F O}

Article history:

Received 24 May 2013

Accepted 7 August 2013

Available online 20 August 2013

\section{Keywords:}

Forming

Plastic behavior

Normal stress

Strain path

\begin{abstract}
A B S T R A C T
In this study, an approach based on the modified Marciniak-Kuczynski (M-K) method for computation of an extended stress-based forming limit curve (FLC) is presented. The extended stress-based FLC is built based on equivalent plastic stress versus mean stress. This curve has some advantages in comparison with the conventional FLC. This new criterion is much more strain path independent than the conventional FLC. The effect of strain path on the predicted extended stress-based FLC is reexamined. For this purpose, two types of pre-straining on the sheet metal have been loaded. Moreover, the plane stress state assumption is not adopted in the current study. The influence of a through-thickness compressive normal stress is also investigated theoretically. The verifications of the theoretical FLCs are performed by using some available published experimental data.
\end{abstract}

(c) 2013 Elsevier Ltd. All rights reserved.

\section{Introduction}

A conventional Forming Limit Curve (FLC) in terms of major and minor strains is applied to predict necking in sheet metal forming processes [1]. This curve can be obtained experimentally and numerically [2].

Marciniak and Kuczynski [3] presented the most well-known method (e.g., the M-K method) to calculate sheet metal forming limits. They assumed that an initial inhomogeneity in the thickness of the material was existent, and they assessed plastic instability phenomenon using two-zone model. In literature, there are many researches (e.g., see [4-7]) which used the M-K method to obtain the FLC.

Panich et al. [4] presented experimental and numerical analyses of Forming Limit Diagram (FLD) and Forming Limit Stress Diagram (FLSD) for two Advanced High Strength Steel (AHSS) sheets grade DP780 and TRIP780. In this study, initially, the forming limit curves were experimentally determined by means of the Nakazima forming test. Subsequently, analytical calculations of both FLD and FLSD were carried out based on the M-K model. Additionally, the FLSDs were calculated using the experimental FLD data for both investigated steels. Different yield criteria, namely, von Mises,

\footnotetext{
* Corresponding author at: School of Mechanical Engineering, College of Engineering, University of Tehran, P.O. Box 11155-4563, Tehran, Iran. Tel.: +98 21 61114026; fax: +98 2188013029.

E-mail addresses: raminhashemi@ut.ac.ir, ramin61hashemi@yahoo.com (R. Hashemi), cabrinia@ut.ac.ir (K. Abrinia).
}

Hill's 48, and Barlat2000 (Yld2000-2d) were applied for describing plastic flow behavior of the AHS steels. Both Swift and modified Voce strain hardening laws were taken into account. Hereby, influences of the constitutive yield models on the numerically determined FLDs and FLSDs were studied regarding to those resulted from the experimental data. The obtained stress based forming limits were significantly affected by the yield criterion and hardening model. It was found that the forming limit curves calculated by the combination of the Yld2000-2d yield criterion and Swift hardening law were in better agreement with the experimental curves. Finally, hole expansion tests were conducted in order to verify the different failure criteria. It was shown that the stress based forming limit curves could more precisely describe the formability behavior of both high strength steel sheets than the strain based forming limit curves.

Assempour et al. [5] presented a methodology for prediction of the FLSD and reexamine the effect of strain path on prediction of FLSD. The methodology is based on the M-K model. For calculation of sheet metal limiting strains and stresses, a numerical approach using the Modified Newton-Raphson with globally convergence method has been used. The evaluation of the theoretical results has been performed by using the published experimental data for ST12 low carbon steel alloy.

Butuc et al. [6] developed a detailed study on the stress-based forming limit criterion (FLSD) during linear and complex strain paths. The calculation of stress-based forming limits based on experimental strain data was performed by using the method proposed by Stoughton [7]. By applying several combinations of 


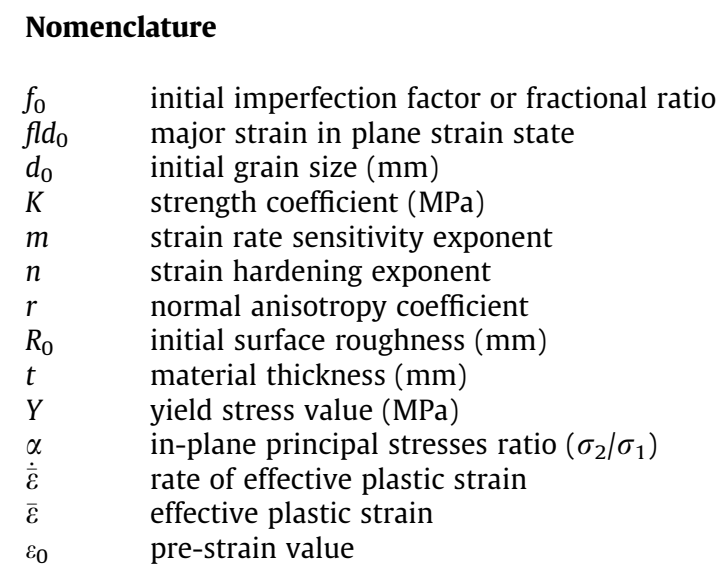

$f_{0}$

fld $_{0}$

$d_{0}$

K

$m$

$n$

$r$

$R_{0}$

$t$

Y

$\alpha$

$\dot{\bar{\varepsilon}}$

$\bar{\varepsilon}$

$\varepsilon_{0}$

\author{
initial imperfection factor or fractional ratio \\ major strain in plane strain state \\ initial grain size $(\mathrm{mm})$ \\ strength coefficient (MPa) \\ strain rate sensitivity exponent \\ strain hardening exponent \\ normal anisotropy coefficient \\ initial surface roughness $(\mathrm{mm})$ \\ material thickness $(\mathrm{mm})$ \\ yield stress value (MPa) \\ in-plane principal stresses ratio $\left(\sigma_{2} / \sigma_{1}\right)$ \\ rate of effective plastic strain \\ effective plastic strain \\ pre-strain value
}

$\begin{array}{ll}d \bar{\varepsilon} & \text { effective plastic strain increment } \\ d \varepsilon & \text { strain increment tensor } \\ d \varepsilon_{1}, d \varepsilon_{2}, d \varepsilon_{3} & \text { strain increments in the material coordinates } \\ d \varepsilon_{n}, d \varepsilon_{n n}, d \varepsilon_{n t} & \begin{array}{l}\text { strain increments in the groove coordinates } \\ \text { groove angle between the groove coordinates and }\end{array} \\ \begin{array}{ll}\text { the material coordinates } \\ \sigma_{1}, \sigma_{2}, \sigma_{3}\end{array} & \begin{array}{l}\text { principal stress components } \\ \sigma_{n n}, \sigma_{n t}, \sigma_{t t}\end{array} \\ \begin{array}{l}\text { in-plane stress components in the groove } \\ \text { coordinates }\end{array} \\ \bar{\sigma}_{Y} & \begin{array}{l}\text { effective stress obtained from hardening law (MPa) } \\ \text { effective stress obtained from yield function (MPa) }\end{array} \\ \bar{\sigma}_{y} & \end{array}$

different constitutive equations on the required plastic calculation, an analysis on the experimental forming stress limits was performed. The necking phenomenon was simulated by $\mathrm{M}-\mathrm{K}$ model using a more general code for predicting the forming limits. The selected materials were a bake-hardened steel (BH steel) and an AA6016-T4 aluminum alloy. Several yield criteria such as Von Mises isotropic yield function, quadratic and non-quadratic criterion of Hill's 1948 [8] and the advanced Barlat Yld96 yield function were used to show the influence of the constitutive law incorporated in the analysis on the stress-based forming limits. The effect of the hardening model on the FLSD was analyzed by using two hardening laws, namely Swift law and Voce law. The influence of work hardening coefficient, strain rate sensitivity and the balanced biaxial yield stress on the theoretical FLSD was also presented. The effect of strain path changes on the stress-based forming limits was analyzed. Some relevant remarks about stress-based forming limit criterion concept were presented.

The effects of strain path on the FLCs were examined by applying various bilinear strain paths (non-proportional loading histories). The FLCs are strain path dependent and strain path has an influence on the FLC and therefore is not valid for formability evaluation of sheet metal forming processes undergoing non-proportional loading paths (e.g., see $[5,6]$ ). To obtain a criterion with less strain path-dependency, a stress-based FLC or forming limit stress curve (FLSC) was presented and implemented to detect necking [9]. The prediction of the sheet metal strain and stress limits generally presumes plane stress states. This hypothesis is only valid for processes with negligible out of plane stresses such as an open die stamping. Thus, a few works $[10,11]$ have studied the influence of through-thickness compressive normal stress on the prediction of the FLC. It is reported that the sheet metal limit strains increase when the through-thickness compressive normal stress increases.

Recently, Simha et al. [12] presented an extended stress-based FLC that could be used to predict the onset of necking in sheet metal loaded under non-proportional load paths, as well as under three-dimensional stress states. They transformed the conventional strain-based FLC into the stress-based FLC advanced by Stoughton [7]. Then, they converted the obtained curve into the extended stress-based FLC, which was characterized by the two invariants, mean stress and equivalent stress. Assuming that the stress states at the onset of necking under plane stress loading were equivalent to those under three-dimensional loading, the extended stress-based FLC was used in conjunction with finite element computations to predict the onset of necking during tubular hydroforming. Hydroforming of straight and pre-bent tubes of EN-AW 5018 aluminum alloy and DP 600 steel were considered. Experiments carried out with these geometries and alloys were described and modeled using finite element computations. These computations, in conjunction with the extended stress-based FLC, allowed quantitative predictions of necking pressures; and these predictions were found to agree to within $10 \%$ of the experimentally obtained necking pressures. The computations also provided a prediction of final failure location with remarkable accuracy. In some cases, the predictions using the extended stressbased FLC showed some discrepancies when compared with the experimental results, and the paper addressed potential causes for these discrepancies.

However, in reference [12], Simha and his co-workers did not examine the strain path independency of their new curve. They also assumed that the formability curve in stress space was not affected by a compressive $\sigma_{3}$, where $\sigma_{3}$ acted in the through-thickness direction. This assumption is not adopted in the current study.

Since necking in some processes such as hydroforming can occur at locations where in addition to the in-plane stresses a through thickness compressive normal stress acts, the plane stress assumption is not proper for these processes. Thus, in this work, the effect of normal stress is considered on the prediction of the extended FLC.

In this paper, the modified $\mathrm{M}-\mathrm{K}$ theory for the computation of the extended stress-based FLC is developed. Two types of prestraining on the sheet metal have been loaded to reexamine the effect of strain path on the predicted extended stress-based FLC. The effect of a through-thickness compressive normal stress on the extended stress-based FLC is also investigated theoretically. The verifications of the computed results are done by using some available published experimental data.

\section{Theoretical analysis}

\subsection{Review of the $M-K$ method}

In this work, the $\mathrm{M}-\mathrm{K}$ analysis (e.g., see $[13,14]$ ) is represented to compute sheet metal strain and stress forming limits considering the effects of a thorough-thickness normal stress and strain path. The basic assumption of this theory is considering a narrow groove in the sheet metal surface (e.g., a geometrical inhomogeneity). It is assumed that there is a defect region and material thickness of this region is slightly smaller than a safe zone. The zone with a nominal thickness (safe zone) is denoted by "a" and the defect zone is denoted by "b". This geometrical inhomogeneity leads to the plastic instability (localized necking) in the sheet. The schematic representation of the M-K theory is illustrated in Fig. 1. To model the defect zone, a fractional ratio is defined $\left(f=t_{b} / t_{a}\right)$, where " $t_{a}$ " denotes the thickness of the zone with the nominal 


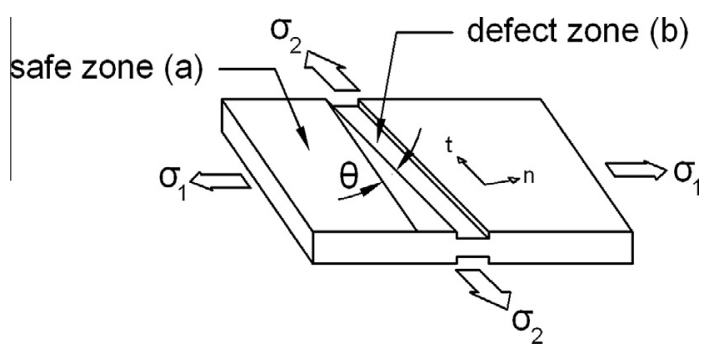

Fig. 1. Schematic of the $M-K$ analysis.

thickness and " $t_{b}$ " denotes the thickness of the defect zone. As a result of loading, the defect region grows and gradually develops into a neck. Ultimately, necking initiates from the defect zone.

\subsection{Methodology of calculation}

By applying a small strain increment $\left(d \bar{\varepsilon}_{a}=0.0001\right)$ in the safe zone, the loading begins and the effective strain or equivalent strain, $\bar{\varepsilon}_{a}$, will be obtained. The effective strain in each step will be determined by:

$\bar{\varepsilon}_{\text {new }}^{a}=\bar{\varepsilon}_{\text {old }}^{a}+d \bar{\varepsilon}^{a}$

Using hardening law (e.g., Swift hardening law), the equivalent stress is obtained. Having the equivalent stress, the through-thickness compressive normal stress $\left(\sigma_{3}\right)$, the stress ratio $\left(\alpha=\sigma_{2} / \sigma_{1}\right)$ and the yield function (e.g., Hill's quadratic yield criterion [8]), $\sigma_{1}^{a}$ and $\sigma_{2}^{a}$ are determined and the stress matrix in the safe zone is as follows (e.g., in principal coordinate system):

$\left[\sigma^{a}\right]_{x y z}=\left[\begin{array}{ccc}\sigma_{1}^{a} & 0 & 0 \\ 0 & \sigma_{2}^{a} & 0 \\ 0 & 0 & \sigma_{3}^{a}\end{array}\right]$

To calculate the stress matrix in the groove coordinates (e.g., see Fig. 1), rotation matrix is applied:

$T=\left[\begin{array}{ccc}\cos \theta & \sin \theta & 0 \\ \sin \theta & \cos \theta & 0 \\ 0 & 0 & 1\end{array}\right]$

$[\sigma]_{n t z}=T^{T}[\sigma]_{x y z} T$

$[d \varepsilon]_{n t z}=T^{T}[d \varepsilon]_{x y z} T$

Now all sheet metal strains and stresses in zone with the nominal thickness can be obtained by using incompressibility condition and flow rule. Thus, all the required information of zone with the nominal thickness is obtained and then all the strains and stresses in the defect zone should be computed. In the defect zone, unknown parameters are $\sigma_{n n}^{b}, \sigma_{n t}^{b}, \sigma_{t t}^{b}, \varepsilon_{n n}^{b}, \varepsilon_{n t}^{b}, \varepsilon_{t t}^{b}$ and $\varepsilon_{3}^{b}$ but applying incompressibility condition and flow rule, they are reduced to $\varepsilon_{n n}^{b}, \varepsilon_{n t}^{b}, \varepsilon_{t t}^{b}, d \bar{\varepsilon}^{b}$ [15]. To find these unknowns, four equations are needed. These equations are the strain compatibility equation, energy equation, and two force equilibrium equations:

$\frac{d \varepsilon_{t t}^{b}}{d \varepsilon_{t t}^{a}}-1=0$

$\frac{d \varepsilon_{n n}^{b} \sigma_{n n}^{b}+d \varepsilon_{t t}^{b} \sigma_{t t}^{b}+2 d \varepsilon_{n t}^{b} \sigma_{n t}^{b}+d \varepsilon_{z z}^{b} \sigma_{z z}^{b}}{d \bar{\varepsilon}^{b} \bar{\sigma}^{b}}-1=0$

$f \frac{\sigma_{n n}^{b}}{\sigma_{n n}^{a}}-1=0$

$f \frac{\sigma_{n t}^{b}}{\sigma_{n t}^{a}}-1=0$

where $f$ changes according to: $f=\exp \left(\varepsilon_{3}^{b}-\varepsilon_{3}^{a}\right)$

To solve this set of non-linear equations, a numerical method is required [16]. In this study, Newton-Raphson method is used as follows:

A typical problem gives $\mathrm{N}$ functional equations in terms of variables $x_{i}, i=1,2, \ldots, N$ to be equal to zero [16]:

$F_{i}\left(x_{1}, x_{2}, \ldots x_{N}\right)=0, i=1,2, \ldots, N$

Let $x$ denotes the vector of values of $x_{i}$ and $F$ denotes the vector of functions $F_{i}$. In the neighborhood of $x$, each of functions $F_{i}$ can be expanded in Taylor series [16]:

$F_{i}(x+\delta x)=F_{i}(x)+\sum_{j=1}^{N} \frac{\delta F_{i}}{\delta x_{j}} \delta x_{j}+O\left(\delta x^{2}\right)$

In matrix notation, this relation can be rewritten as:

$F(x+\delta x)=F(x)+J \cdot \delta x+O\left(\delta x^{2}\right)$

where $J$ is the Jacobin matrix:

$J_{i j}=\frac{\partial F_{i}}{\partial x_{j}}$

By setting $F(x+\delta x)=0$ and neglecting terms of the order of $\delta x^{2}$, a set of equations are obtained:

$J . \delta x=-F$ or $\delta x=-J^{-1} F$

Then the solution vector can be determined,

$X_{\text {new }}=x_{\text {old }}+\delta x$

and the procedure is iterated until convergence.

In this numerical procedure, $d \bar{\varepsilon}^{b}$ and $d \bar{\varepsilon}^{a}$ are computed in each step and compared with each other, if $d \bar{\varepsilon}^{b} / d \bar{\varepsilon}^{a}>10$ the necking is occurred $[13,14]$ and limiting strains $\left(\varepsilon_{1}^{a}, \varepsilon_{2}^{a}\right)$, are saved. This numerical process is repeated for various groove angles and stress ratios until the whole limit strains are obtained.

A major problem to the theoretical methods such as the $\mathrm{M}-\mathrm{K}$ theory is that the computed FLCs are so susceptible to the inhomogeneity coefficient, $f$ [17]. Therefore, several methods (e.g., see [18]) have been suggested to calibrate the computed FLD. In this study, to reduce this sensitivity, $f$ is related to grain size and surface roughness of the sheet (e.g., see $[17,18])$. Thus, $f$ is given:

$f=f_{0} \exp \left(\varepsilon_{3}^{b}-\varepsilon_{3}^{a}\right)$

$f_{0}=\frac{t_{0}^{a}-2\left(R+k d_{0}^{0.5} \varepsilon_{e}^{b}\right)}{t_{0}^{a}}$

In these relations, $d_{0}$ is the initial grain size, $k$ is the material constant and $R$ is the initial surface roughness of the sheet.

\subsection{FLD computation under non-linear strain path}

To obtain sheet metal forming limits under non-proportional loadings, two types of pre-straining have been loaded on the sheet metal prior to obtaining its FLD. A bilinear strain path is made up of two stages. At each stage the loading is linear and described by the following relation $[5,6]$ :

$$
\left\{\begin{array}{lll}
\rho=\rho_{1} & \text { if } \quad & \varepsilon_{1}^{\mathrm{a}} \leqslant \varepsilon_{1 s}^{a} \\
\rho=\rho_{2} & \text { if } & \varepsilon_{1}^{\mathrm{a}}>\varepsilon_{1 s}^{a}
\end{array}\right.
$$

where $\rho_{1}$ and $\rho_{2}$ specified the strain ratios that are applied and $\varepsilon_{1 s}^{a}$ is the pre-strain value. 
2.4. FLD computation considering the existence of compressive normal stress

For any arbitrary through-thickness compressive normal stress (e.g., $\sigma_{3}$ ) and for any arbitrary stress ratio of $\alpha$ (e.g., $\alpha=\sigma_{2} / \sigma_{1}$ ), the components of principal stresses $\sigma_{1}$ and $\sigma_{2}$ are described as the following [19]:

$\sigma_{1}=\frac{-B+\sqrt{\left(B^{2}-4 A C\right)}}{2 A}$

where,

$$
\begin{aligned}
& A=\alpha^{2}+1+r(1-\alpha)^{2} \\
& B=-2 \sigma_{3}(\alpha+1) \\
& C=2 \sigma_{3}^{2}-\frac{2}{3}(2+r) \bar{\sigma}_{y}^{2}
\end{aligned}
$$

In equation 16 , " $r$ " is the normal anisotropy coefficient.

\section{Yield function and hardening law}

Based on general form of Hill's quadratic yield criterion [8], the equivalent stress is given as follows:

$\bar{\sigma}_{y}=\sqrt{\frac{3}{2}}\left[\frac{F\left(\sigma_{2}-\sigma_{3}\right)^{2}+G\left(\sigma_{3}-\sigma_{1}\right)^{2}+H\left(\sigma_{1}-\sigma_{2}\right)^{2}}{F+G+H}\right]^{1 / 2}$

where $F, G, H$ are coefficients of Hill's 1948 yield function [8]. Assuming the existence of planar isotropy, the yield criterion can be specified as follows [8]:

$\bar{\sigma}_{y}=\sqrt{\frac{3}{2}}\left[\frac{\left(\sigma_{2}-\sigma_{3}\right)^{2}+\left(\sigma_{3}-\sigma_{1}\right)^{2}+r\left(\sigma_{1}-\sigma_{2}\right)^{2}}{[2+r]}\right]^{1 / 2}$

For sheet metals, the $r$ values are usually obtained for three various directions of loading in-plane (e.g., $0^{\circ}, 45^{\circ}, 90^{\circ}$ to the rolling direction) and the normal $r$-value is taken to be the average (e.g., $\left.r=\frac{r_{0}+2 r_{45}+r_{90}}{4}\right)$.

A simplified hardening law (Power law relation) [20] is given as follows:

$\bar{\sigma}_{Y}=K\left(\varepsilon_{0}+\bar{\varepsilon}\right)^{n}(\dot{\bar{\varepsilon}})^{m}$

where $\bar{\sigma}_{Y}$ denotes the effective stress, $\varepsilon_{0}, \bar{\varepsilon}$ are the pre-strain and the effective strain respectively, $n$ is the strain hardening exponent, $K$ is the strength constant, $\dot{\bar{\varepsilon}}$ denotes the effective strain rate and $m$ is the strain-rate sensitivity exponent.

\section{Extended stress-based FLC}

As mentioned earlier and reexamined here, the FLCs are strain path dependent. So this curve cannot be applied to analyze sheet metal forming process taken under non-linear strain paths. Recently, the extended stress-based FLC has been presented in [12] and it was claimed that this curve was much more strain path independent than the conventional FLC. Moreover, it was assumed that the formability curve in stress space was not affected by a compressive $\sigma_{3}$, where $\sigma_{3}$ acted in the through-thickness direction. This assumption is not adopted in the current study. The effect of the through-thickness compressive normal stress on the extended stress-based FLC is also investigated theoretically in this paper. As one can be seen in results and discussion section, the throughthickness compressive normal stress has influence on the extended stress-based FLC.

This extend FLC is constructed based on effective stresses (equivalent stresses) versus mean stresses at the onset of localized necking [12]. The equivalent stress is calculated using Hill's yield criterion [8] (e.g., see Eqs. (17) and (18)) and the mean stress is calculated as follows:

$\sigma_{\text {hyd }}=\frac{\sigma_{1}+\sigma_{2}+\sigma_{3}}{3}$

As with conventional forming limit curve, the extended FLC divide the condition for the onset of necking, under which supposed to be invulnerable from failure [12]. However, this extended FLC is much more strain path independent than the conventional FLC and applicable to any forming paths under non-proportional loadings.

The extended stress-based FLC can be implemented into finite element numerical simulations to analyze and design the sheet metal forming process. Since finite element software such as ABAQUS or ANSYS can compute the strains incrementally in each element, therefore the mean stress and the equivalent stress in each element can be derived at every increment of deformation. Ultimately, the equivalent stresses and corresponding mean stresses for the entire strain path of each element can be determined and the deformation process can be analyzed by comparing the equivalent stresses vs. the mean stresses for the final strain increment with the extended stress-based FLC.

\section{Results and discussion}

Firstly, verifications of theoretical FLCs were done by comparing results of the present work with some published experimental data under the plane stress conditions and when the through-thickness compressive normal stress was considered. Furthermore, the computed FLCs under non-linear strain paths were validated. The mechanical properties of these materials have been presented in Tables 1 and 2 presents material constants used in the inhomogeneity coefficient function, $f_{0}$. Eq. (13) is used to calibrate the FLCs of ST12 low carbon steel and for the other materials used in this study; the imperfection factor $f_{0}$ was used as a factor for calibration (e.g., see $[13,14,21])$. Then the effects of the through-thickness compressive normal stress and strain path changes on the extended stress-based FLC were investigated theoretically.

\subsection{Verifications of theoretical results}

To evaluate theoretical results obtained in this study, several experimental data are selected. Fig. 2a illustrates a comparison between the experimental data [22] and the theoretical FLC obtained under proportional loading and under plane stress state $\left(\sigma_{3}=0\right)$. The predicted FLC is in reasonable agreement with the experimental data for as-received ST12 low carbon steel. Fig. 2b illustrates the computed and experimentally determined FLCs [23] for the ST14 low carbon steel obtained under proportional loading and under plane stress state. This figure illustrates a suitable agreement between the predicted FLC and the experimental FLC determined without pre-straining and for the plane stress conditions. To determine the FLCs experimentally in references [22,23], the outof-plane Nakazima test [23] was performed. This test involves two stages: fixing the sample using circular die rings as the blank-holder, and stretching the sample using a hemispherical punch. A hydraulic press was employed to provide the required force. In reference [23], to obtain the FLC and generate all required

Table 1

Mechanical properties.

\begin{tabular}{lllllll}
\hline Material & $K(\mathrm{MPa})$ & $n$ & $m$ & $\varepsilon_{0}$ & $r$ & $t(\mathrm{~mm})$ \\
\hline ST12 [22] & 238 & 0.3 & 0.01 & 0.01 & 1.21 & 2.5 \\
ST14 [23] & 505 & 0.26 & - & - & 1.20 & 1.5 \\
AA6011 [24] & 254.9 & 0.265 & - & - & 0.574 & 1.86 \\
STKM-11A [25] & 1500 & 0.15 & - & - & 2.14 & 1.4 \\
\hline
\end{tabular}


Table 2

Material constants using in the relation 13 [22]

\begin{tabular}{lllll}
\hline Material & $k$ & $d_{0}(\mathrm{~mm})$ & $R(\mathrm{~mm})$ & $t_{0}(\mathrm{~mm})$ \\
\hline ST12 & 0.104 & 0.025 & 0.006 & 2.5 \\
\hline
\end{tabular}

FLCs, ST12, f0=0.995

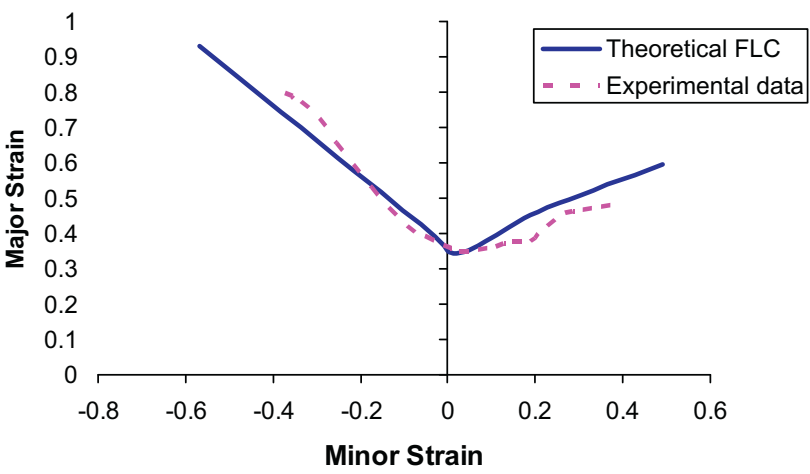

(a)

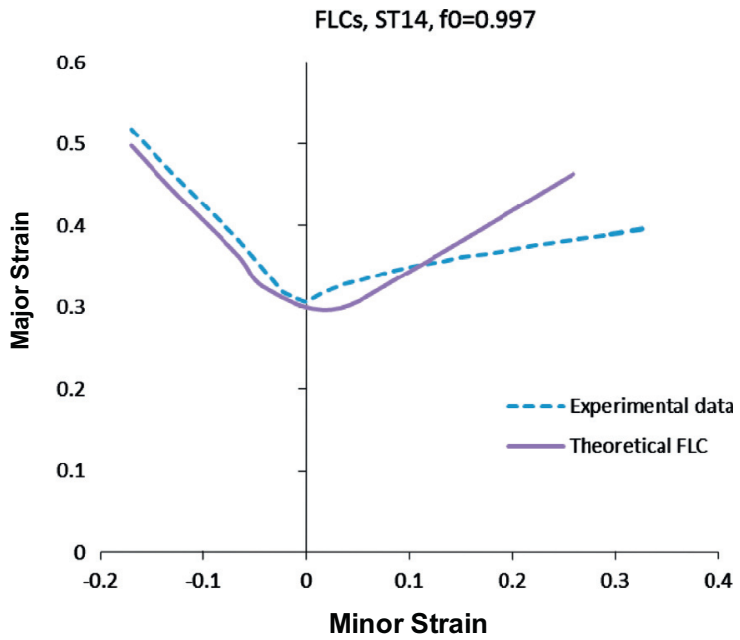

(b)

Fig. 2. FLCs under proportional loading and plane stress condition, (a) ST12 low carbon steel and (b) ST14 low carbon steel.

strain states, different sample geometries and lubricants were used.

The theoretical and experimental FLCs [22] of ST12 low carbon steel determined under non-proportional loading paths (e.g., bilinear loadings) are shown in Figs. 3 and 4. Fig. 3a and Fig. 3b illustrate the computed and experimental FLCs after tensile pre-strain levels of $\varepsilon_{1 s}^{a}=0.1$ and $\varepsilon_{1 s}^{a}=0.15$ respectively. Fig. 4 shows the computed and experimental FLCs after biaxial pre-strain levels of $\varepsilon_{1 s}^{a}=0.08$. The predicted FLCs for ST12 low carbon steel after pre-straining in uniaxial and tension parallel to the prior rolling direction (e.g., Figs. 3 and 4) show almost good correlation with experiments.

To verify the computed FLCs with the existence of a throughthickness compressive normal stress $\left(\sigma_{3} \neq 0\right)$, some published experimental data are chosen. Fig. 5a shows the computed and experimental FLCs [24] for AA6011 aluminum tubes. In reference [24], the corresponding experimental process was tube hydroform-
FLCs, ST12, Pre-straining, f0 =0.995

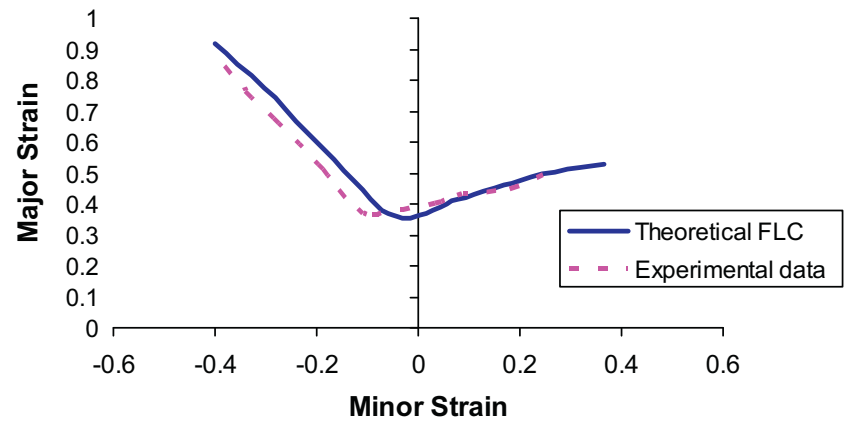

(a)

FLCs, ST12, Pre-straining, f0 =0.995

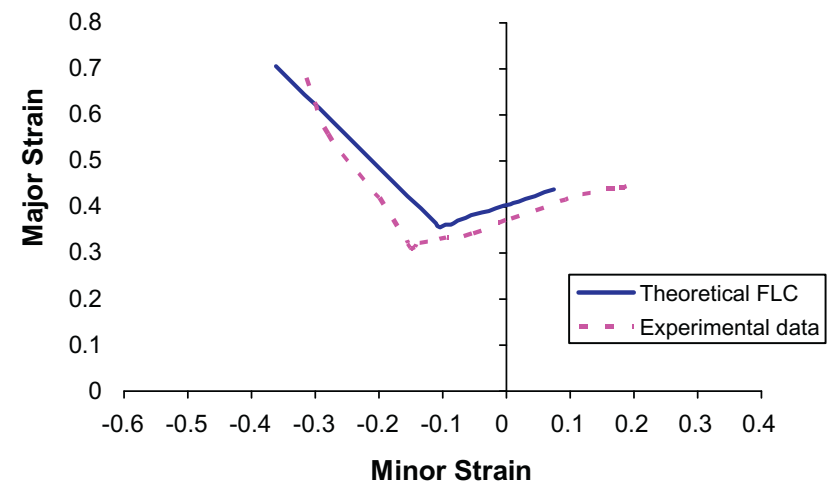

(b)

Fig. 3. (a) FLCs after uniaxial tensile pre-strain of $\varepsilon_{1 \mathrm{~s}}^{a}=0.1$ and (b) FLCs after uniaxial tensile pre-strain of $\varepsilon_{1 s}^{a}=0.15$.

FLCs, ST12, Pre-straining, f0 $=0.995$

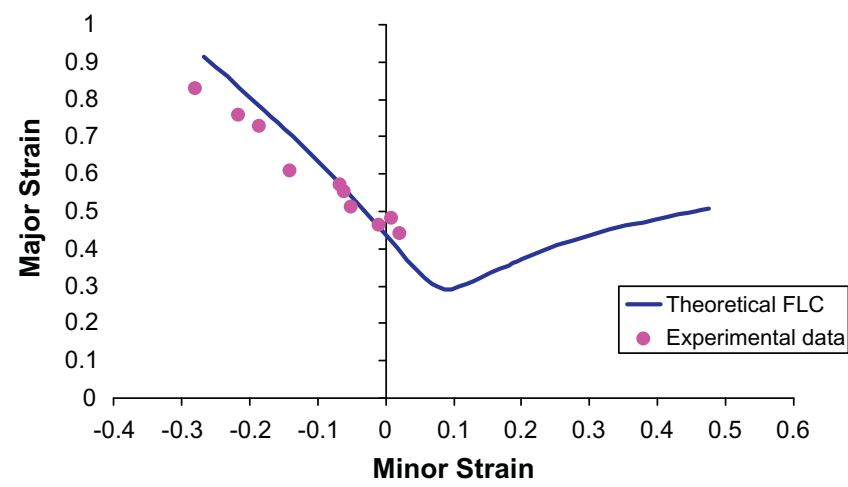

Fig. 4. FLCs after equi-biaxial pre-strain of $\varepsilon_{1 s}^{a}=0.08$.

ing with up to $-12 \mathrm{MPa}$ internal pressure. Fig. 5b, illustrates the computed and experimental FLCs [25] for STKM-11A steel alloy with the existence of a through-thickness compressive normal stress of $-56 \mathrm{MPa}$. Kim et al. [25] carried out a series of bulge tests and the tubes were supported between a lower and an upper die. In the present numerical study, since the die supported the outer surface, thus the same value of internal pressure -56 MPa has been applied. In reference [25], there were no experimental data for the right hand side of the FLC.

However, the difference between theoretical results and experiment may be due to errors in measuring the strains; because the 
FLCs, AA6111, S3=-6 MPa, f0 $=0.997$

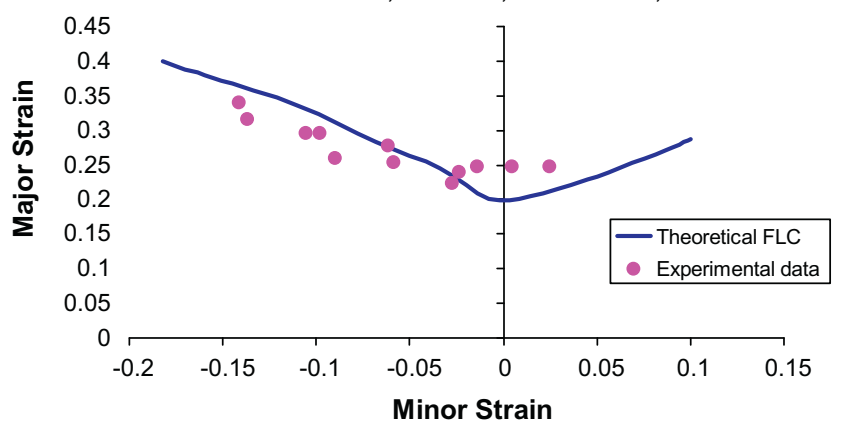

(a)

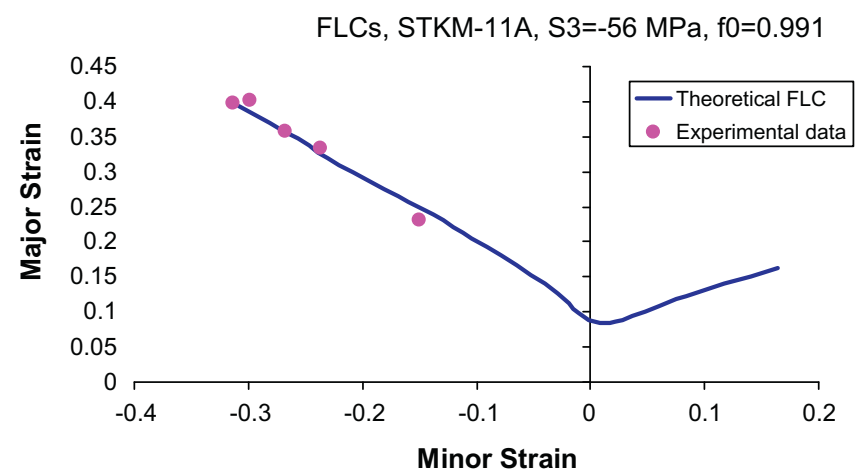

(b)

Fig. 5. (a) FLCs for AA6011 with the existence of a through-thickness compressive normal stress $\left(\sigma_{3}=-6 \mathrm{MPa}\right)$ and (b) FLCs for STKM-11A with the existence of a through-thickness compressive normal stress $\left(\sigma_{3}=-56 \mathrm{MPa}\right)$.

experimental data used to evaluate the present approach were determined by the conventional circle grid analysis method. Therefore, the errors originating from this method inevitably led to some variability in the results.

\subsection{Effect of strain path on the extended FLCS}

To study the influence of strain path on the extended FLC, based on the present method a series of FLCs with and without prestrains are generated (e.g., see Figs. 2a, 3 and 4). ST12 low carbon steel is selected in this example. As illustrated in Fig. 2a, a conventional FLC with linear strain path is determined theoretically. Then, as illustrated in Fig. 3a and b, two FLCs are computed under 0.10 and 0.15 uniaxial pre-tensions. The limit strains are shifted to left and increase as pre-strain level increase. A FLC is also computed in the same way under 0.08 equi-biaxial pre-strain as illustrated in Fig. 4. In this mode of pre-straining, limit strains are shifted to right and decrease as pre-strain levels increase. This trend is consistent with experimental testing data (see [22]).

As expected, Figs. 3 and 4 illustrate the strain path dependent nature of the conventional FLCs. Thus, recently, a new stress-based FLC has been introduced [14] and this new criterion is strain path independent unlike the conventional FLCs.

Now, the strain path independency of an extended stress-based FLC is reexamined by converting the conventional FLCs in Figs. 2a, Fig. 3 and 4 (or Fig. 6a) to the extended FLCs. As shown in Fig. 6b, all the FLCs at different loading paths are degenerated to one single curve. So the present results confirm that the extended stressbased FLC is strain path independent unlike the conventional FLC.

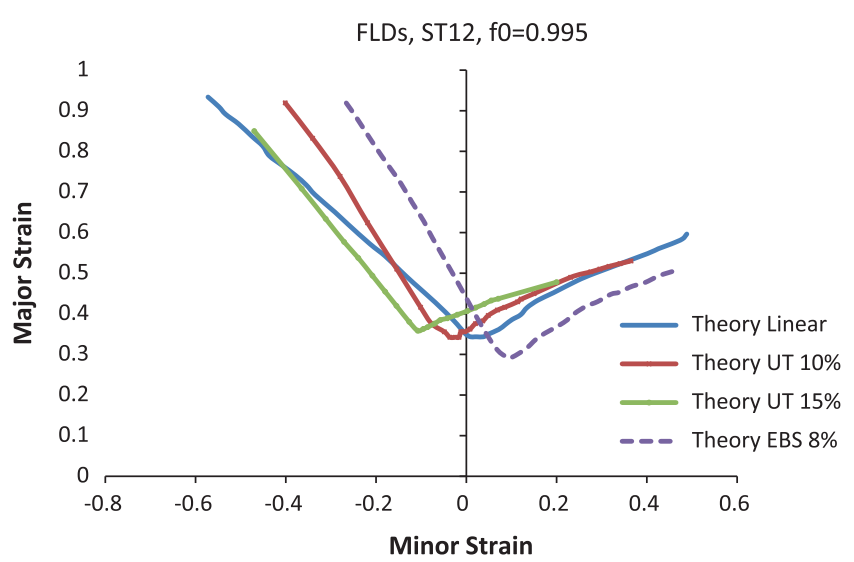

(a)

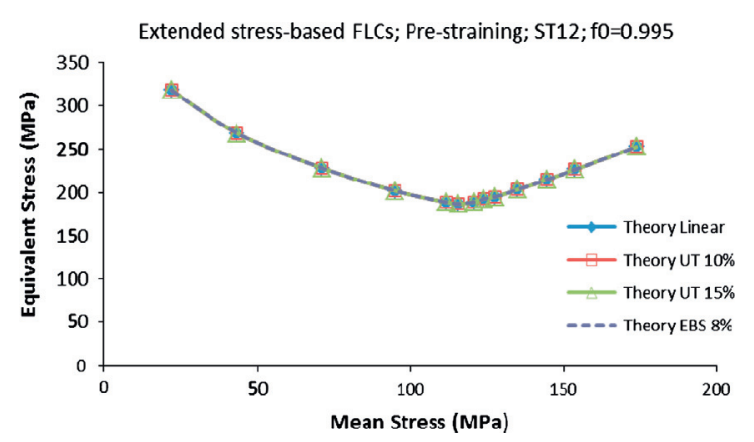

(b)

Fig. 6. (a) The theoretical FLCs for different loading paths and (b) The theoretical extended stress-based FLCs for different loading paths (present work).

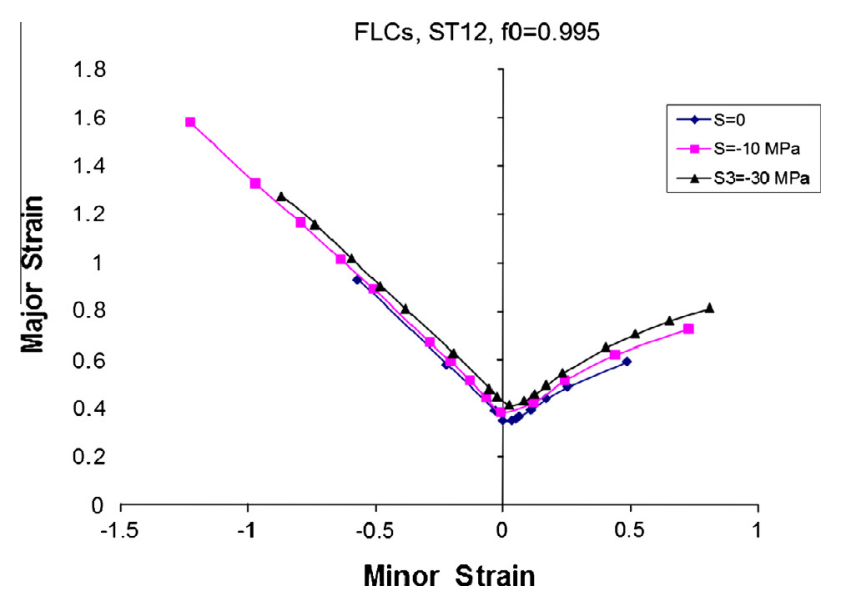

Fig. 7. The influence of the through-thickness compressive normal stress on prediction of the traditional FLCs.

\subsection{Effect of the through-thickness normal stress on the extended FLCS}

Since the effect of a through-thickness compressive normal stress cannot be neglected in some processes such as hydroforming because of high applied pressure (see $[10,11])$. Therefore, the effects of a through-thickness compressive normal stress component on the FLCs, FLSCs and the extended stress-based FLC are illustrated in Figs. 7-9 respectively. ST12 low carbon steel is chosen in this work. Fig. 7 shows that with increasing the absolute value of the through-thickness compressive normal stress, the FLC 


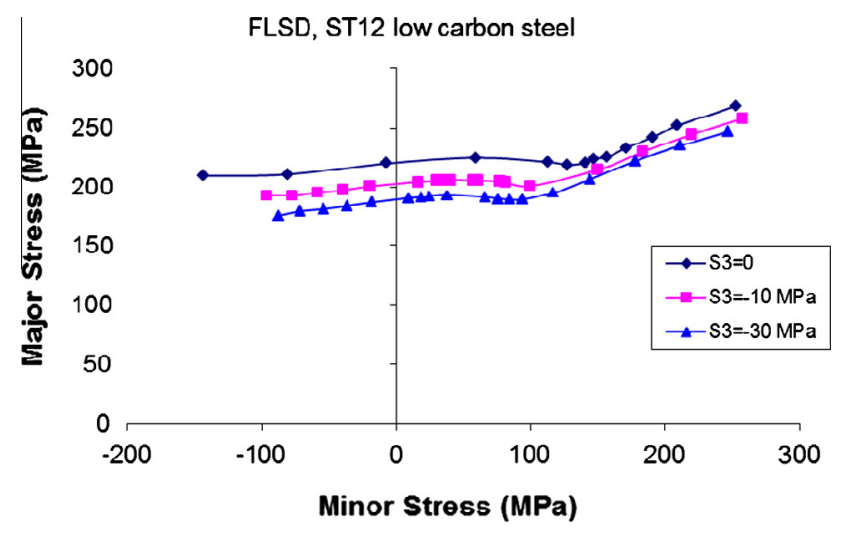

Fig. 8. The influence of the through-thickness compressive normal stress on prediction of the stress-based FLCs.

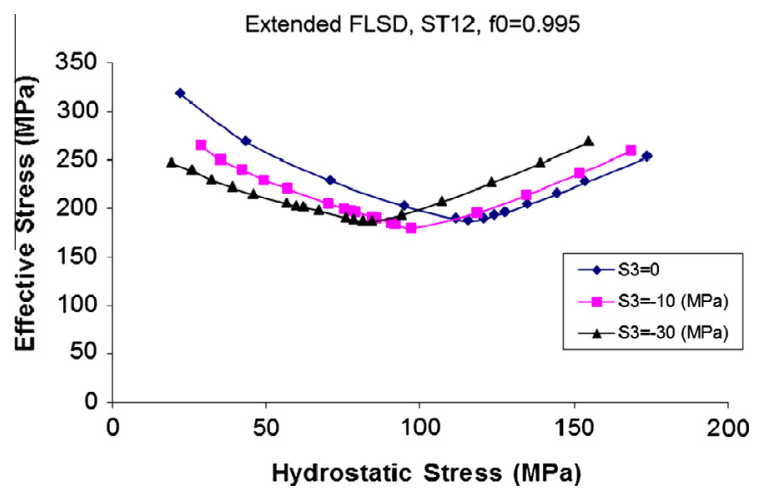

Fig. 9. The influence of the through-thickness compressive normal stress on prediction of the new extended FLCs.

Table 3

Percentage of effective stress $\left(\bar{\sigma}_{y}\right)$ variation " $\xi$ " by increasing the absolute value of the normal stress " $\sigma_{3}$ " for the extended FLC of ST12 (initial value of $\sigma_{3}$ is zero).

\begin{tabular}{lll}
\hline Mode of forming & $\sigma_{3}(\mathrm{MPa})$ & $\xi$ \\
\hline Uniaxial tension & $\left\{\begin{array}{l}0 \\
-10\end{array}\right.$ & -8.56 \\
Plane strain & $\left\{\begin{array}{l}0 \\
-30\end{array}\right.$ & -14.41 \\
Equibiaxial stretching & $\left\{\begin{array}{l}0 \\
-10\end{array}\right.$ & -4.76 \\
& $\left\{\begin{array}{l}0 \\
-30\end{array}\right.$ & -1.43 \\
& $\left\{\begin{array}{l}0 \\
-10\end{array}\right.$ & 2.78 \\
& $\left\{\begin{array}{l}0 \\
-30\end{array}\right.$ & 6.35 \\
\hline
\end{tabular}

level goes up and also displaces slightly to the right. This trend is in agreement with $[10,11]$. Fig. 8 illustrates opposite behavior for the FLSCs compared with the FLCs.

As mentioned earlier, Simha et al. [12] assumed that the formability curve in stress space was not affected by a compressive $\sigma_{3}$, where $\sigma_{3}$ acted in the through-thickness direction. This assumption is not adopted in the current study and Fig. 9 shows that the through-thickness compressive normal stress has effect on the extended stress-based FLC. Thus, this factor cannot be neglected in determination of the extended stress-based FLC, especially for hydroforming processes.
Table 3 illustrates percentage of the theoretical equivalent stress variation " $\xi$ " for different values of the normal stress $\left(\sigma_{3}\right)$ for the extended FLC of ST12. The parameter $\xi$ is described as follows:

$$
\xi=\frac{\bar{\sigma}_{y} \text { with existence of normal stress }-\bar{\sigma}_{y} \text { plane stress }}{\bar{\sigma}_{y} \text { plane stress }} \times 100
$$

\section{Conclusions}

Since necking in some processes such as hydroforming can occur at locations where in addition to the in-plane stresses a through thickness compressive normal stress acts, the plane stress assumption is not proper for these processes. This assumption is not adopted in the current study. In this article, the M-K method was represented for prediction of the extended stress-based FLC. The effect of normal stress is considered on the prediction of the extended stress-based FLC. The obtained results showed that the through-thickness compressive normal stress has effect on the extended stress-based FLC. Furthermore, the strain path-independency of this curve was reexamined. The obtained results showed that the extended FLC is much more strain path independent than the conventional FLC.

\section{References}

[1] Liu Ianguang, Liu Wei, Xue Wei. Forming limit diagram prediction of AA5052 polyethylene/AA5052 sandwich sheets. Mater Des 2013;46:112-20.

[2] Ko Dae-Cheol, Cha Seung-Hoon, Lee Sang-Kon, Lee Chan-Joo, Kim Byung-Min. Application of a feasible formability diagram for the effective design in stamping processes of automotive panels. Mater Des 2010;31(3):1262-75.

[3] Marciniak Z, Kuczynski K. Limit strains in the process of stretch-forming sheet metal. Int J Mech Sci 1967:9:609-20.

[4] Panich Sansot, Barlat Frédéric, Uthaisangsuk Vitoon, Suranuntchai Surasak, Jirathearanat Suwat. Experimental and theoretical formability analysis using strain and stress based forming limit diagram for advanced high strength steels. Mater Des 2013;51:756-66.

[5] Assempour A, Hashemi R, Abrinia K, Ganjiani M, Masoumi E. A methodology for prediction of forming limit stress diagrams considering the strain path effect. Comput Mater Sci 2009;45:195-204.

[6] Butuc MC, Gracio JJ, Barata da Rocha A. An experimental and theoretical analysis on the application of stress-based forming limit criterion. Int J Mech Sci 2006;48:414-29.

[7] Stoughton TB. A general forming limit criterion for sheet metal forming. Int J Mech Sci 2000;42:1-27.

[8] Hill R. A theory of the yielding and plastic flow of anisotropic metals. Proc R Soc Lond A 1948:193:281-97.

[9] Hashemi R, Abrinia K, Assempour A. The strain gradient approach to predict necking in tube hydroforming. J Manuf Processes 2013;15(1):51-5.

[10] Smith LM, Averill RC, Lucas JP, Stoughton TB, Matin PH. Influence of transverse normal stress on sheet metal formability. Int J Plast 2003;19:1567-83.

[11] Allwood JM, Shouler DR. Generalized forming limit diagrams showing increased forming limits with non-planar stress states. Int J Plast 2009:25:1207-30.

[12] Simha CHM, Gholipour J, Bardelcik A, Worswick MJ. Prediction of necking in tubular hydroforming using an extended stress-based forming limit curve. Transaction of the ASME. J Eng Mater Technol 2007;129(1):36-48.

[13] Hashemi R, Assempour A, Masoumi E. Implementation of the forming limit stress diagram to obtain suitable load path in tube hydroforming. Mater Des 2009;30(9):3545-53.

[14] Hashemi R, Faraji G, Abrinia K, Dezaji AF. Application of the hydroforming strain- and stress-limit diagrams to predict necking in metal bellows forming process. Int J Adv Manuf Technol 2010;46(5-8):551-61.

[15] Assempour A, Khakpour Nejadkhaki H, Hashemi R. Forming limit diagrams with the existence of through-thickness normal stress. Comput Mater Sci 2010;48(3):504-8.

[16] Ganjiani M, Assempour A. Implementation of a robust algorithm for prediction of forming limit diagrams. J Mater Eng Perform 2008:17(1):1-6.

[17] Safikhani AR, Hashemi R, Assempour A. Some numerical aspects of necking solution in prediction of sheet metal forming limits by strain gradient plasticity. Mater Des 2009:30:727-40.

[18] Ghazanfari A, Assempour A. Calibration of forming limit diagrams using a modified Marciniak-Kuczynski model and an empirical law. Mater Des 2012;34:185-91.

[19] Hashemi R. Extension of sheet metal forming limits theory considering strain path and normal stress effects. PhD Dissertation. School of Mechanical Engineering. University of Tehran; 2013. 
[20] Safikhani AR, Hashemi R, Assempour A. The strain gradient approach fo determination of forming limit stress and strain diagrams. Proc IMechE Part B J Eng Manuf 2008;222(4):467-83.

[21] Faraji G, Hashemi R, Mashhadi MM, Dezaji AF. Hydroforming limits in metal bellows forming process. Mater Manuf Processes 2010:25(12):1413-7.

[22] Shakeri M, Sadough A, Dariani BM. Effect of pre-straining and grain size on the limit strains in sheet metal forming. Proc IMechE Part B J Eng Manuf $2000 ; 214: 821-7$
[23] Hashemi R, Ghazanfari A, Abrinia K, Assempour A. Forming limit diagrams of ground St14 steel sheets with different thicknesses. SAE Int J Mater Manuf 2012;5:60-4

[24] Hwang YM, Lin YK, Chuang HC. Forming limit diagrams of tubular materials by bulge tests. J Mater Process Technol 2009;209:5024-34.

[25] Kim J, Kang SJ, Kang BS. A prediction of bursting failure in tube hydroforming processes based on ductile fracture criterion. Int J Adv Manuf Technol 2003;22:357-62. 